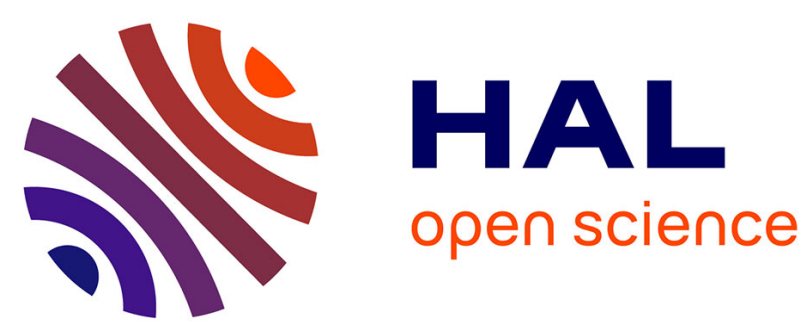

\title{
Analysis of Opportunistic Localization Algorithms Based on the Linear Matrix Inequality Method
}

Francesco Zorzi, Andrea Bardella, Tanguy Pérennou, Guodong Kang, Andrea Zanella

\section{- To cite this version:}

Francesco Zorzi, Andrea Bardella, Tanguy Pérennou, Guodong Kang, Andrea Zanella. Analysis of Opportunistic Localization Algorithms Based on the Linear Matrix Inequality Method. The Second International Workshop on Mobile Opportunistic Networking ACM/SIGMOBILE MobiOpp 2010, Feb 2010, Pisa, Italy. 4p. hal-00452360v2

\section{HAL Id: hal-00452360 https://hal.science/hal-00452360v2}

Submitted on 3 Feb 2010

HAL is a multi-disciplinary open access archive for the deposit and dissemination of scientific research documents, whether they are published or not. The documents may come from teaching and research institutions in France or abroad, or from public or private research centers.
L'archive ouverte pluridisciplinaire HAL, est destinée au dépôt et à la diffusion de documents scientifiques de niveau recherche, publiés ou non, émanant des établissements d'enseignement et de recherche français ou étrangers, des laboratoires publics ou privés. 


\title{
Analysis of Opportunistic Localization Algorithms Based on the Linear Matrix Inequality Method
}

\author{
Francesco Zorzi ${ }^{\dagger}$, Andrea Bardella ${ }^{\dagger}$, Tanguy Pérennou*, \\ Guodong Kang ${ }^{\S \ddagger}$, Andrea Zanella ${ }^{\dagger}$ \\ † Dipartimento di Ingegneria dell'Informazione, Università degli Studi di Padova, Italy \\ * LAAS-CNRS \& ISAE, Université de Toulouse, France \\ $\S$ TeSA \& ISAE, Université de Toulouse, France \\ ¥ Northwestern Polytechnical University, Xi'an, China \\ E-mail: \{zorzifra, bardella, zanella\}@dei.unipd.it, \{perennou, gkang\}@isae.fr
}

\begin{abstract}
With the increasing spread of use of mobile devices there is a growing demand for location-aware services in a wide variety of contexts. Yet providing an accurate location estimation is difficult when considering cheap off-the-shelf mobile devices, particularly in indoors or urban environments. In this paper we define and compare different localization algorithms based on an opportunistic paradigm. In particular, we focus on range-free and range-based localization techniques that are based on the solution of a Linear Matrix Inequalities (LMI) problem. The performance achievable with this approach is analyzed in different scenarios, through extensive simulation campaign. Results show that LMI-based schemes, especially the range-based one, are potentially capable of yielding very accurate localization even after a limited number of opportunistic exchange, though performance is rather sensitive to the accuracy of the other nodes' self-localization and to the randomness of the radio channel.
\end{abstract}

\section{Categories and Subject Descriptors}

C.2.2 [Computer-Communication Networks]: Network Protocols

\section{General Terms}

Performance, Design

\section{Keywords}

Localization, LMI, Wireless Networks, Opportunistic

\section{INTRODUCTION}

Mobile devices are getting more and more popular: they are simultaneously getting smaller and more powerful. This spread of use has attracted a lot of interest for location-aware services and systems.

Permission to make digital or hard copies of all or part of this work for personal or classroom use is granted without fee provided that copies are not made or distributed for profit or commercial advantage and that copies bear this notice and the full citation on the first page. To copy otherwise, to republish, to post on servers or to redistribute to lists, requires prior specific permission and/or a fee.

MobiOpp '10, February 22-23, 2010, Pisa, Italy.

Copyright 2010 ACM 978-1-60558-925-1/10/02 ...\$10.00.
Researchers have already proposed a number of solutions to perform accurate positioning in situations where the GPS is not available. They generally imply an infrastructure with reference positions and techniques measuring received signal strength (RSS), time of arrival, time difference of arrival or angle of arrival to derive positions using triangulation or multilateration.

Alternatively, some authors have proposed solutions based on the collective and opportunistic use of all available position estimations. In such a context, our previous work [7] has shown that a node (user) completely unaware of its own position but receiving inaccurate position estimations from other nodes (peers) can still compute accurate positions. Peers opportunistically deliver inaccurate estimations of their own positions when they can communicate with the user.

There are several ways to compute an accurate position based on inaccurate position estimations from peers. One of the most promising approaches is based on Linear Matrix Inequalities (LMI) and was introduced by Doherty et al. [2] In previous work [7], we proposed a range-free LMI scheme where the inequalities took into account self-positioning errors as well as a constant maximum communication range. This paper is the continuation of that work and has the following contributions (see Section 3).

First, we propose a range-based LMI scheme in which the Received Signal Strength (RSS) from each peer is used to estimate an upper bound of the user-peer distance.

Second, we propose a Hybrid approach for LMI scheme that trades off computational effort for accuracy by selecting subsets of inequalities before solving the system.

The proposed scheme and improvements are evaluated in Section 4 through Matlab simulations, in order to investigate the localization accuracy when varying the number of available contact opportunities in different scenarios.

\section{SYSTEM MODEL}

This section describes the underlying assumptions and models of our study.

\subsection{Communication model}

We assume every node in the network is equipped with a common wireless communication interface that is used for (opportunistic) data exchange. Radio propagation is modeled through the classic path loss and shadowing model [3], so that the power $\Gamma_{r}(d)$ received at distance $d$ from the trans- 
mitter is given by

$$
\Gamma_{r}(d)=\Gamma_{t}+K-10 \eta \log _{10}\left(\frac{d}{d_{0}}\right)+\psi,
$$

where $\Gamma_{t}$ is the transmission power [in dB], $K$ is a unitless constant that depends on the environment, $d_{0}$ is the reference distance in meters for the antenna far field, and $\eta$ is the so-called path loss coefficient that depends on the environment. Finally, $\psi$ is a Normal random variable, with zero mean and variance $\sigma_{\psi}^{2}$, that describes the long term fading (or shadowing) effect.

We assume that opportunistic data exchange is enabled only if the received power is above a certain threshold $\Gamma_{t h} \geq$ $\Gamma_{\min }$, where $\Gamma_{\min }$ is the minimum power level that guarantees successful packet decoding with high probability. The threshold $\Gamma_{t h}$ defines the so-called nominal opportunistic range $R_{t h}$ given by

$$
R_{t h}=d_{0} 10^{\frac{\Gamma_{t}+K-\Gamma_{t h}}{10 \eta}} .
$$

In principle, opportunistic data exchange shall be enabled only between nodes within distance $R_{t h}$. However, the random fluctuations of the received power due to the shadowing term $\psi$ in (1) makes the actual range $R_{o p p}$ for opportunistic data exchange a log-normal distributed random variable, given by

$$
R_{o p p}=R_{t h} 10^{\frac{\psi}{10 \eta}} .
$$

We observe that $R_{\text {opp }}$ can take any (positive) value, though the probability of large values goes rapidly to zero. Since the opportunistic localization algorithms described later in this paper require to fix an upper bound on the opportunistic range, we define $R_{\max }$ as the distance under which opportunistic data exchange occurs with probability 0.99 , that is to say

$$
P\left[R_{o p p} \leq R_{\max }\right]=0.99 .
$$

We hence assume nodes are uniformly distributed in a circle with radius $R_{\max }$ centered in the User and, according to (1), we admit opportunistic data exchange between Peer and User only when the received power at the User is above $\Gamma_{t h}$.

\subsection{Ranging model}

We assume that, during an opportunistic data exchange, nodes can perform some sort of ranging, i.e., they can estimate their distance from some physical measurements, such as the received signal strength indication (RSSI) or the Time of Arrival (ToA) of the radio signals. In our study, we consider two possible ranging scenarios, namely range-free, and range-based.

\subsubsection{Range-free scenario}

In this case, we consider a dummy ranging technique that provides a constant range estimate

$$
\hat{d}=R_{t h},
$$

irrespective of the actual distance between transmitter and receiver (provided that the received power is above the opportunistic threshold $\Gamma_{t h}$ ). The maximum ranging error is hence equal to $\epsilon_{\text {rang }}^{\max }=R_{\max }-R_{t h}$, with high probability.

\subsubsection{Range-based scenario}

In this case we assume an RSSI-based ranging technique, which uses the value of signal power $\Gamma_{r}$ measured by the receiver to provide a Maximum-likelihood estimate $\hat{d}\left(\Gamma_{r}\right)$ of the real distance $d$ between transmitter and receiver. The estimate is obtained by reversing the path-loss equation (1) once neglected the shadowing term, which yields

$$
\hat{d}=d_{0} 10^{\frac{\Gamma_{t}+K-\Gamma_{r}}{10 \eta}}=d 10^{\frac{\psi}{10 \eta}} .
$$

From (6) we note that the accuracy of the RSSI-based ranging estimation depends on the shadowing term $\psi$ and is more accurate for short distances. As for the opportunistic range, given a distance $d$, we also define an upper bound $d_{\text {max }}$ on the RSSI-based ranging estimate, such that

$$
P\left[\hat{d} \leq d_{\max }\right]=0.99 .
$$

Hence, the ranging error for a range estimate $\hat{d}$ with high probability is limited to $\epsilon_{\text {rang }}^{\max }=d_{\max }-\hat{d}$.

\subsection{Self-positioning model used by peers}

We assume that peer nodes have "native" self-positioning capabilities, provided by some (non opportunistic) scheme. Accordingly, we denote by $P_{i}$ and $\widehat{P}_{i}$ the real and the selfestimated position of the $i$-th peer. For simplicity, we assume that the estimation error $e_{i}=\left\|P_{i}-\widehat{P}_{i}\right\|$ can be modeled as the module of a 2-dimensional Gaussian Random Variable $[x, y]$, with zero mean and variance $\sigma_{l o c}^{2}$. Consequently, the self-localization distance error $\epsilon=\sqrt{x^{2}+y^{2}}$ is a Rayleigh-distributed random variable with parameter $\sigma_{l o c}$. Hence, the parameter $\sigma_{l o c}$ defines the accuracy of the native self-localization mechanism supported by the node. For simplicity, in each simulation we assume that all Peers have the same $\sigma_{l o c}$. Once again, it is practical to set an upper bound $\epsilon_{l o c}^{\max }$ on the self-localization error of Peers, such that

$$
P\left[\epsilon \leq \epsilon_{\text {loc }}^{\max }\right]=0.95 .
$$

\section{OPPORTUNISTIC LOCALIZATION ALGORITHMS}

As mentioned, User resorts to opportunistic localization to infer its geographical position. To this end, User opportunistically collects information from passing-by Peers. Information consists of i) $\widehat{P}_{i}$, which is the current position estimate of Peer $i$, provided by self-localization system; ii) $\epsilon_{l o c}^{\max }$, which is the maximum self-localization error, as given by (8); iii) a ranging estimate $\hat{d}$ and maximum ranging error $\epsilon_{\text {rang }}^{\max }$, which depends on the ranging technique adopted by User, as specified in Sec 2.2. User is assumed to remain still while collecting information for opportunistic localization. Then, it performs one of the opportunistic localization algorithms described below to get an estimate $\widehat{P_{u}}$ of its real position $P_{u}$.

\subsection{Centroid algorithm}

The Centroid algorithm [1] estimates User's position as

$$
\widehat{P_{u}}=\sum_{i=1}^{N} \widehat{P}_{i},
$$

where $N$ is the total number of Peers encountered during the opportunistic localization phase.

Centroid does not rely upon any ranging information, but only on Peers self-localization estimations. This scheme is really simple, not requiring any knowledge about the channel and self-positioning error models. On the other hand, its performance strongly depends on the spatial distribution of 
Peers. Although this localization algorithm is very basic, it provides a useful benchmark to compare the performance of more advanced schemes.

\subsection{LMI localization algorithm}

The main approach for opportunistic localization considered in this paper is based on the solution of Linear Matrix Inequality (LMI) problems. Basically, for each Peer $i$ involved in opportunistic data exchange, User writes the following inequality

$$
\left\|P_{u}-\widehat{P}_{i}\right\| \leq R_{i}
$$

where $\|\cdot\|$ denotes the euclidian distance, and $R_{i}$ is the maximum admissible distance between User and Peer $i$ given by $R_{i}=\hat{d}+\epsilon_{\text {rang }}^{\max }+\epsilon_{\text {loc }}^{\max }$. The set of inequalities (10) collected during the opportunistic phase confines User's position in the area covered by the intersection of the circles centered in $\widehat{P}_{i}$ and having radius $R_{i}$, for $i=1,2, \ldots, N$. The User's position is then estimated by choosing a point $\widehat{P_{u}}$ within this area, possible at maximum distance from the area border. This problem can be formulated as a Linear Matrix Inequality (LMI) problem [2], which can be solved with standard techniques.

We observe that, due to the approximate bounding (4), (7), and (8), it is possible that User receives messages from Peers farther than $R_{i}$, though with very low probability. In this case, the circles intersection may be empty and the LMI problem becomes unfeasible. When this event happens, we solve again the LMI problem after increasing the value of $R_{i}$.

\subsection{Hybrid LMI-Centroid algorithm}

Although there are efficient numerical methods to solve an LMI, the resolution time rapidly grows with the number of inequalities in the system. To cope with this problem, we propose a Hybrid LMI algorithm (HLMI) that combines the LMI and Centroid techniques. Basically, to limit the computational cost of LMI we partition the $N$ inequalities in $K=N / L$ groups of $L$ inequalities, thus obtaining $K$ temporary estimates $\widehat{P_{u}}(k)$, with $k=1,2, \ldots, K$. Then, we apply the barycentric technique to obtain the final estimate $\widehat{P_{u}}$ as

$$
\widehat{P_{u}}=\sum_{k=1}^{K} \widehat{P_{u}}(k) .
$$

The hybrid scheme makes it possible to trade off localization accuracy for computational cost.

\section{PERFORMANCE ANALYSIS}

In this Section, we analyze the results provided by the three opportunistic localization algorithms, namely Centroid, LMI and Hybrid LMI, in different scenarios. For all the experiments, we consider the path-loss channel parameters $\Gamma_{t}=0 \mathrm{dBm}, \eta=1.74, K=-36 \mathrm{~dB}, d_{0}=0.1 \mathrm{~m}$ and $R_{t h}=10 \mathrm{~m}$. For each scenario, we ran 60 experiments to obtain enough statistical accuracy. We calculated the confidence intervals for each scenario. Having less than $10 \mathrm{~cm}$ in the ideal scenario and less than $25 \mathrm{~cm}$ when adding sources of error, we do not show the intervals overlapped on the curves in the graphs for the sake of clarity.

We first analyze the effects of the different random components, i.e. the self-localization accuracy and the shadowing of the channel, on the behavior of the different algorithms.
As expcected, adding self-localization error to the Peers, the localization performance decreases. Nonetheless, rangebased LMI shows good performance if the uncertainty of the self-localization is not too high, i.e. $\sigma_{l o c}=2$, while the range-free approach is more sensitive to this error. The reason is that the circles drawn around the Peers are larger due to the increases of $R_{i}$ and centered in the wrong point. The Centroid algorithm shows no significant differences. This is due to the fact that self-localization errors of Peers are assumed to have zero mean, so that if Peers are uniformly distributed in the area, after a sufficient number of contacts the errors will compensate.

Increasing $\sigma_{\psi}$, the randomness of the signal propagation brings along ranging errors and unpredictable opportunistic data exchange range, since communication can occur even at distances larger than $R_{t h}$, whereas it may happen that nodes within $R_{t h}$ do not communicate. With $\sigma_{\psi}=2$, range-based LMI performs better than Centroid, whereas increasing the randomness of the channel using $\sigma_{\psi}=4$, LMI has quite poor accuracy, keeping the localization accuracy below the Centroid only for the first 5 contacts. In this scenario, for a larger number of contacts, the probability that the LMI problem becomes unfeasible grows, so that it is necessary to relax the constraints as explained in Sec. 3.2, thus worsening performance. The capacity of range-based algorithm to use small circles, when the contact is performed with close nodes, increases the estimate accuracy, limiting the intersection area close to the User. On the contrary the range-free algorithm has very large circles that means quite poor localization performance.

In summary, the LMI approach, in particular the rangebased, is potentially capable of yielding good localization, though it suffers from high computational complexity and performance degradation for large number of contacts and unreliable channels. In the following we hence investigate the performance of the hybrid LMI approach, which may alleviate some of these problems.

In Fig. 1, the three red solid lines show the performance of Hybrid LMI with range-free technique, for different values of $L$, with deterministic radio channel and unreliable Peers' self-localization. First, it is possible to notice that, with $L=3$, the Hybrid algorithm achieves basically the same performance of Centroid. With $L=10$ the Hybrid LMI performs slightly better than Centroid. The blue dashed lines in Fig. 1 show the same results for range-based Hybrid LMI scheme. We see that, also in this case, the Hybrid approach yields better performance than pure-based approach when the number of contacts increases, in particular when self-localization error of Peers increases.

When channel conditions become harsh, the problem of unfeasibility of the LMI solution for large numbers of inequalities has a strong impact. Therefore it is more convenient to use a small $L$, both in range-free and range-based LMI to achieve better results, as shown in Fig. 2. Where the classic LMI range-based has worse performance than the Centroid, the HLMI achieves better performance than Centroid, and in addition it also decreases the computational complexity. The same behavior can be seen for the range-free HLMI: the less the $L$, the more the accuracy, approaching very close to the Centroid performance.

\section{RELATED WORK}

Self-localization problem has been investigated in a number of papers. Most common localization methods consist in measuring the power of the received RF signal (RSSI), 


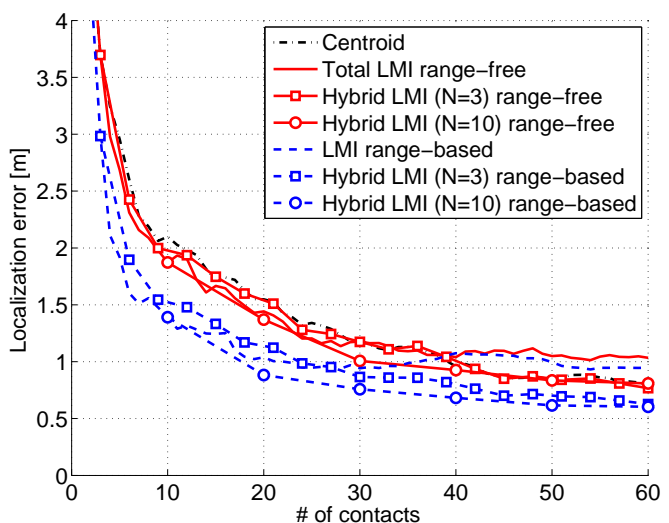

Figure 1: Localization performance comparison between range-free and range-based Hybrid LMI with different values of $L$, with $\sigma_{\psi}=0 \mathbf{d B}, \sigma_{l o c}=2 \mathbf{m}$.

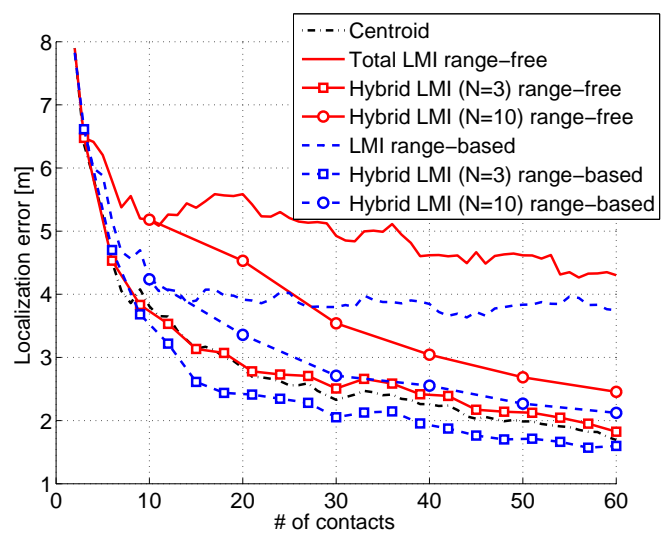

Figure 2: Localization performance comparison between range-free and range-based Hybrid LMI with different values of $L$, with $\sigma_{\psi}=4 \mathrm{~dB}, \sigma_{l o c}=2 \mathrm{~m}$.

the Time of Arrival (ToA) or the Angle of Arrival (AoA) of the RF signals from the beacons. In this way, every node estimates a set of distances from the beacons and, then, guesses its position by means of lateration and triangulation techniques or by using statistical estimation methods. Overviews of localization techniques based on RSSI and ToA measurements can be found in [6]. An approach similar to LMI system is the Min-Max algorithm [4, 5], in which squares are drawn around beacon nodes and the final estimation is chosen inside the intersection. The main difference is that in our study, we do not consider a pre-planned beacon scenario, but a mobile and opportunistic one. In [2] Doherty et al. pioneered the use of semidefinite programming (SDP) methods in the localization problem. The problem is considered as a bounding problem containing several convex geometric constraints mathematically representated as linear matrix inequalities (LMI). The mechanism proposed in this paper is based on this approach, taking into estimation errors and introducing a barycentric improvement over time.

\section{CONCLUSION}

In this paper, we addressed the problem of the localization of strayed node, called User, by means of opportunistic data exchange with passing by nodes, called Peers, which are instead capable of self-localization. We studied two main localization schemes, namely Centroid, in which User estimates its position as average of Peers' position estimations, and LMI that takes into account a connectivity model and a ranging model to provide a bound on the distance between Peer and User.

We simulated different scenarios, varying the randomness of the signal propagation and the self-localization accuracy of Peers. Results revealed that, in favorable scenarios, the LMI localization achieves very good accuracies, especially for the range-based scheme. On the contrary, when the signal propagation is random or Peers self-localization is not reliable, LMI performance degrades faster than Centroid's one. Furthermore, we observed that the LMI localization performance worsens, compared to the Centroid technique, when the number of contacts exceeds a given threshold with unreliable channels, because the constraints considered in the LMI problem become unfeasible and need to be relaxed. It is worth to notice that the symmetric choice of nodes distribution gives an advantage to the performance of the Centroid algorithm, whereas it suffers in a scenario where ndoes are deployed asymmetrically with respect to the User. Hence, we analyzed the Hybrid LMI algorithm that splits the LMI problem in subproblems of $L$ inequalities that are resolved independently and, then, computes the barycenter of the different solutions. In this case, localization accuracy strongly depends on the choice of $L$, though a suitable tuning of this parameter is effective in counteracting the performance loss due to constraints relaxation in difficult scenarios. Furthermore, Hybrid LMI algorithm dramatically reduces the computational effort.

\section{ACKNOWLEDGMENTS}

This work was partly supported by the European Commission in the framework of the FP7 Network of Excellence in Wireless COMmunications NEWCOM++ (contract n. 216715), by French ANR Telecoms Project FIL and by "Fondazione Cassa di Risparmio Padova e Rovigo" under the project "A large scale wireless sensor network for pervasive city-wide ambient intelligence."

\section{REFERENCES}

[1] N. Bulusu, J. Heidemann, and D. Estrin. Gps-less low cost outdoor localization for very small devices. IEEE Personal Communications Magazine, October 2000.

[2] L. Doherty, L. El Ghaoui, and K. S. J. Pister. Convex position estimation in wireless sensor networks. In Proc. of IEEE INFOCOM, pages 1655-1663, Anchorage, AK, USA, April 2001.

[3] A. Goldsmith. Wireless Communications. Cambridge University Press, New York, NY, USA, 2005.

[4] K. Langendoen and N. Reijers. Distributed localization in wireless sensor networks: a quantitative comparison. Comput. Networks, 43(4):499-518, 2003.

[5] X. Nguyen and T. Rattentbury. Localization algorithms for sensor networks using RF signal strength. Technical report, University of California at Berkeley, May 2003.

[6] N. Patwari, J. N. Ash, S. Kyperountas, A. O. Hero III, R. L. Moses, and N. S. Correal. Locating the nodes: cooperative localization in wireless sensor networks. IEEE Signal Processing Magazine, July 2005.

[7] F. Zorzi, G. Kang, T. Pérennou, and A. Zanella. Opportunistic localization scheme based on linear matrix inequality. In Proc. of WISP 2009, Budapest, Hungary, August 2009. 